\title{
The Impact of Electronic Prescribing on the Professionalization of Community Pharmacists: A Qualitative Study of Pharmacists' Perception
}

\author{
Aude Motulsky ${ }^{1}$, Nancy Winslade ${ }^{2}$, Robyn Tamblyn ${ }^{2}$ and Claude Sicotte ${ }^{1}$ \\ ${ }^{1}$ Groupe de recherche interdisciplinaire en santé, Université de Montréal, Montréal, Canada. ${ }^{2}$ Clinical and Health \\ Informatics Research Group, McGill University, Montréal, Canada.
}

Received, October 8, 2007; Revised, March 17, 2008; Accepted March 21, 2008, Published, March 24, 2008.

\begin{abstract}
PURPOSE. To understand how the technology of electronic prescription (e-Rx) can transform the community pharmacist's role through its effects on professionalization. We define professionalization as a pharmaceutical practice centred on clinical activities and made possible by the establishment of professional pharmaceutical services. METHODS. We asked 12 community pharmacists who had participated in an e-Rx pilot project in the Canadian province of Quebec to fill out a qualitative survey on their experience. We then analyzed the pharmacists' perceptions of this new technology using a conceptual framework based on the Davenport typology that presents an exhaustive list of mechanisms, specific to Information Technologies, and thus e-Rx, that can potentially modify information management process and then the role of pharmacists. RESULTS. The pharmacists identified five main mechanisms by which e-Rx could affect the professionalization of community pharmacists: analytical capabilities of the pharmacist and physician, dissemination of knowledge, integration of process tasks, process automation and elimination of intermediaries. These mechanisms can assist pharmacists in exercising their professional judgement by improving the quality of available information and facilitate the execution of prescriptions by improving the quality of orders. E-Rx technology can also strengthen pharmacists' credibility as medication specialists in the eyes of both patients and physicians. Thus, e-Rx can become a collaborative technology to the extent that it improves collaboration between community pharmacists and prescribing physicians. However, the potential benefits of this technology would appear to depend on its characteristics and how prescribing physicians use it. CONCLUSIONS. E$\mathrm{Rx}$ proposes ways of working and communicating that were previously unimaginable. These new possibilities pave the way for transformations that can significantly increase the professionalization of community pharmacists. The results of this study indicate that community pharmacists have a favourable opinion of e-Rx, believing it can be an ally in their professionalization.
\end{abstract}

\section{INTRODUCTION}

The role of the pharmacist has changed radically in the past century. Originally a careful mixer of ointments and medicines, the apothecary became a distributor of drugs with the industrialization of drug production in the second half of the $20^{\text {th }}$ century. In the increasingly commercial context, the practice of community pharmacy came to be centred around the distribution of drugs and no longer their preparation, particularly in North America $(1,2)$. In response to this commercial trend, the profession's elites initiated a clinical shift in an attempt to reposition the pharmacist around the patient and away from the drug (3). This movement led to the appearance of the concept of pharmaceutical care in the 1980s. According to its traditional definition, pharmacists who practice pharmaceutical care assume responsibility for ensuring that patients achieve pharmacotherapeutic objectives that improve the quality of life of their patients $(4,5)$. Over the past twenty years, the training of pharmacists has been revised to produce professionals capable of providing pharmaceutical care. Some have used the term "reprofessionalization" to describe the process by which pharmacists have attempted to re-centre their activities around the patient and the patient's use of drugs, and away from the drug and its distribution (6-8). The professionalization of pharmacists is therefore associated with activities of a clinical nature, as opposed to activities of a technical nature.

In line with these changes, a variety of professional services have been implemented in community pharmacies in the past few decades. These services fall under a variety of categories including therapeutic outcome monitoring, medication management and medication counselling. These initiatives represent an attempt by pharmacists to meet patient's unmet medication related needs, requiring pharmacists to expand their role beyond the distribution of medications and the execution of prescriptions.

Corresponding Author: Aude Motulsky, Groupe de recherche interdisciplinaire en santé, Université de Montréal, Montréal (Québec). Canada, Email: aude.motulsky@umontreal.ca 
Pharmacists use their professional judgement, passing along their knowledge and skills on medications to the prescriber and the patient. An increasing number of studies have shown the clinical and economic benefits of the many professional services offered by community pharmacists, particularly in North America and Europe $(9,10)$. However, studies show that these services are not well established in the majority of community pharmacies; instead, prescription execution remains the main focus of practice (1113). Currently, the scope of practice of the community pharmacist is ill-defined, situated somewhere between two ideal types: the health professional, who is altruist and patient-centred, and the shopkeeper, who is focused on efficiency in drug distribution $(2,7,14)$. The commercial context that surrounds the practice in North America accentuates this ambiguity in the minds of patients, other health professionals, and even pharmacists themselves.

The introduction of electronic prescription (e-Rx) technologies in healthcare organizations may be reshaping the field of community pharmacy. In its more restricted definition, electronic prescribing is a process in which the physician uses a computerbased system to prescribe medications (15). Moreover, advanced e-Rx technologies may include other functionalities to support the various clinicians, including the pharmacists, involved in the medication management process in primary care (see (15) for more details). For example, expert softwares and knowledge database may be included to support physicians in their decision-making (in both clinical and cost aspects); the prescription can be transmitted electronically from the physician to the community pharmacist. Furthermore, e-Rx technology can enables the various clinicians involved in medication management to access complete and up-to-date clinical information on patients, because information from different health professionals can be shared on the network. The result may be improved continuity in patient management (15).

The majority of studies on e-Rx have been carried out in the hospital context and have focused on the prescribing process of physicians, the system's main users (15-17). These studies have revealed both intended and unintended consequences of implementing this technology in healthcare organizations, such as the impact on workflow and roles definition of the various professionals involved in medication management $(18,19)$. For the community pharmacist, these effects have rarely been explored. Some experts believe that by facilitating the prescription execution process, e-Rx could be the stepping stone needed to reconcile the functions of medication distribution and provision of professional services (20). In this sense, e-Rx technology could contribute to the professionalization of community pharmacists by freeing them from a number of the technical tasks associated with prescription execution. However, empirical observations in contexts where e-Rx is being used are rare and have not allowed for any testing of this hypothesis (21). Moreover, some researchers maintain the opposite, asserting that information technologies (ITs) such as e-Rx could in fact lead to a deprofessionalization of pharmacists in several ways: by codifying and democratizing the expert knowledge of pharmacists, e-Rx could reduce their professional territory and competency; by systematizing the clinical decision-making of pharmacists, e-Rx could decrease their autonomy in exercising their professional judgment; and by enabling tight control of the professional practice of pharmacists, e-Rx could increase the use of disciplinary measures (22-24).

To shed light on these issues, we conducted an exploratory case study of 12 pharmacists who had used e-Rx in the actual context of community pharmacy practice. Our objective was to better understand the pharmacists' perceptions of the ways in which e-Rx technology could potentially transform their role through its effects on professionalization. We will first present our analysis model that describes the potential mechanisms through which e-Rx can facilitate or hinder the professionalization of community pharmacists. We will then present our research methods, followed by the results of our analysis of the interviews with community pharmacists. We end with a discussion of the implications of our findings for the professionalization of community pharmacists.

\section{CONCEPTUAL FRAMEWORK}

In order to characterize the effects of e-Rx technology on the professionalization of community pharmacists, we used the typology developed by Davenport $(25,26)$. The Davenport typology includes nine mechanisms by which an IT can transform an information management process. These mechanisms are described in depth, thereby enabling us to describe in a very detailed way the functions of the e-Rx technology with respect to all of the activities of the community pharmacist. We used this typology to investigate the ways in which $\mathrm{e}-\mathrm{Rx}$ technology can modify the medication management process in a community pharmacy, and to identify the potential effects of these changes on the professionalization of community pharmacists. But in order to explore how e-Rx can affect the medication management process, we first needed a model of the process. We chose the model developed by Bell et al. (15), adapting it for the purposes of this study (see Figure 1). 


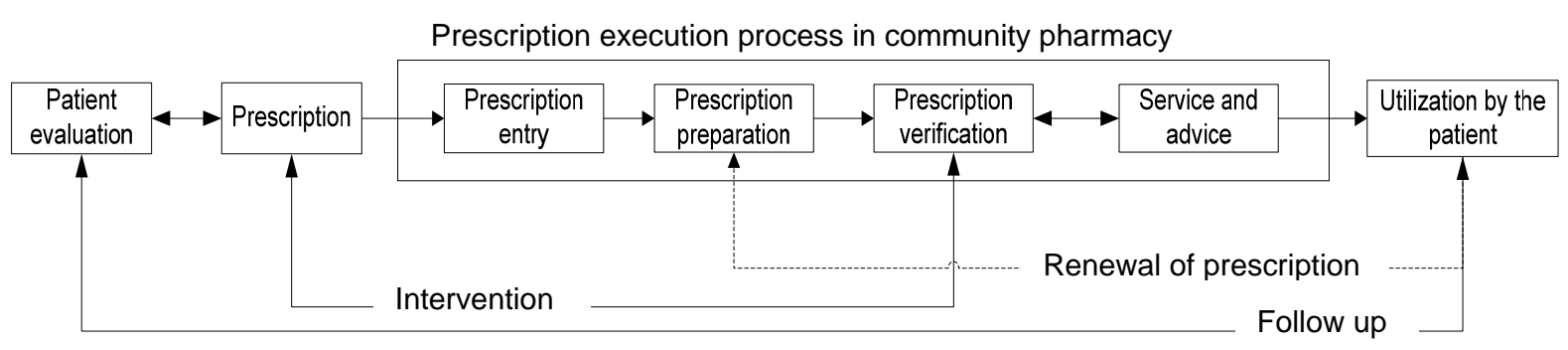

Figure 1. The medication management process. (Adapted from Bell et al. (15))

By breaking down the activities of the community pharmacist in a detailed way, we were able to conceptualize the potential effects of e-Rx on these activities, and deduce the probable consequences on the professional role of the pharmacist. Table 1 gives a detailed description of each mechanism, as well as its potential effects, both positive and negative, on the professionalization of pharmacists. When we tried to conceptualize the effects of e-Rx on the professional role of the community pharmacist, we succeeded in operationalizing seven of the nine mechanisms contained in this typology. This clearly shows the significant potential of e-Rx. The two omitted mechanisms were: the sequential mechanism - Changing process sequence, or enabling parallelism - and the geographical mechanism - Coordinating processes across distances - that would not be possible given the strict legal framework governing the professional activities in community pharmacies.

\section{METHODS}

\section{Background}

An e-Rx technology was implemented as a research project in primary care medical clinics in a large urban area in the Canadian province of Quebec (see (27)). The participating physicians were provided with a computer that was directly connected to the central database of the MOXXI Project. These databases linked information from the Quebec health insurance agency, known as RAMQ, with data from community pharmacies. This meant the physicians had access to a database containing information on the medical problems, medical visits and actual medication consumption of their consenting patients. When a physician entered a prescription, the expert software would propose dosage regimens and check for any medicationrelated problems (alerts) in the patient's file. Also, the physician was required to enter the treatment indication associated with each medication (s)he prescribed. Two copies of the prescription were printed, one for the patient and one for the physician's files, and the prescription was sent electronically to the secure central database. When the patient arrived at a participating pharmacy, the pharmacist downloaded the prescription on the local computerized dispensing system using a code given on the paper prescription. Twenty-five pharmacies downloaded a total of $458 \mathrm{e}-\mathrm{Rxs}$ to their computer system over a 6-month period.

\section{Study procedure}

We conducted a qualitative study of the community pharmacists that participated in the e-Rx project (a total of 70 pharmacists). All of the 25 owner pharmacists of participating pharmacies received a letter explaining the objective of the study and asking them to participate. We then contacted them by phone to set up an appointment for an interview. We met with the first eight pharmacists who agreed to take part. We also solicited the participation of salaried pharmacists who were on duty when we interviewed the owner pharmacists. Since we believed this number was sufficient for a qualitative analysis, we did not take any further steps to recruit more subjects. Furthermore, no new theme emerged from the last interviews, indicating that saturation was reached. A total of 12 pharmacists were interviewed for an overall participation rate of $17 \%$. The pharmacists' experiences with electronic prescribing varied greatly: while six of the pharmacists downloaded $15 \%$ of all e-Rxs generated during the entire 6-month pilot project, the other six pharmacists tried the system but executed a lower number of e-Rxs, with two pharmacies unable to receive electronic transmissions because of technical problems with the network. In the latter two pharmacies, the pharmacists used the patient's paper copy of the prescription since they could not download the electronic version. All of the pharmacists possessed a good understanding of the new system, having notably received full training for the e-Rx technology.

The 12 community pharmacists participated in semi-directed interviews aimed at documenting their perceptions of the new technology implemented in their workplace. We decided to use 
the interview as our data collection method because it enables the researcher to study a little known phenomenon in more depth. The interview consisted of open-ended questions on the influence of e-Rx on the day-to-day work of community pharmacists, on their relationship with patients and physicians, and on the role of the community pharmacist. We chose questions that were sufficiently broad that pharmacists could describe their personal experience as well as their expectations with respect to the technology. We did not want to constrain their answers by using targeted questions on the precise functions of e-Rx. This study was approved by the Research Ethics Board of McGill University.

\section{Analysis}

We recorded the interviews and then transcribed them in their entirety. A deductive content analysis was performed using the software ATLAS.Ti for the coding procedure. We developed our codebook according to Patton's procedure (28); that is, the codes were defined from our open-ended questions and from our initial reading of the interviews. Some additional codes were added during the coding procedure when necessary. We checked the consistency of the codebook by inverse coding after the first codification. This procedure allows the researcher to validate the consistency of the codes by reading all of the quotations associated with a specific code. Every interview was coded twice. We merged the codes into categories of the medication management process and then created comparative tables. The purpose of this step was to compare pharmacists' perceptions about the effect of e-Rx for every activity of the process. Finally, we used the Davenport typology to classify the pharmacists' perceptions according to the different mechanisms. We wrote a preliminary report and sent it to all of the pharmacists interviewed. They sent us their comments, which we reviewed and used to improve the validity of the final analysis.

\section{RESULTS}

Based on their experience in the pilot project, the pharmacists identified five mechanisms by which e-Rx technology can influence their professional role: analytical capacity of the pharmacist and physician, dissemination of knowledge, integration of process tasks, process automation and elimination of intermediaries. Table 2 summarizes the effects associated with these mechanisms and gives representative quotes from the pharmacists interviewed. Our analysis of the potential effects associated with these mechanisms, as perceived by the pharmacists interviewed, revealed that two important dimensions of the pharmacist's role are involved: the exercise of professional judgement and the execution of prescriptions. We will elaborate on the effects of the relevant mechanisms in the following sections, according to these two dimensions.

\section{Exercise of professional judgement}

According to the pharmacists interviewed, the mechanisms most likely to promote their professionalization are those that improve the quality of information available to them when they are exercising their professional judgement. This can be when they are checking a prescription sent in by a physician (analytical capacity and elimination of intermediaries), or when analyzing the patient's file (integration of process tasks). In fact, all of the pharmacists appreciated the fact that e-Rx enabled them to exercise their professional judgement more adequately when verifying a prescription because a greater amount of information was transmitted with the electronic prescription (quote 1, Table 2). The elimination of the patient as an intermediary can also increase the precision of information, thereby increasing the analytical capacity of the pharmacist and facilitating prescription verification (quotes 11.1 to 11.3 , Table 2). The majority of pharmacists predicted that their interventions with patients (quotes 2.1 and 2.2, Table 2) and prescribers (quotes 3.1 and 3.2, Table 2) can become more relevant and meaningful as a result.

The integration of clinical information, resulting from the ability to visualize all of a patient's clinical information, can enable community pharmacists to take a more proactive role in managing the pharmacotherapy of patients (quotes 7.1 and 7.2, Table 2). For example, half of the pharmacists said they would be willing to make a pharmacotherapeutic treatment decision for certain pathologies if the physician transmitted them the patient's relevant clinical data. Similarly, the majority of pharmacists expressed an interest in monitoring the treatment of chronic conditions by adjusting medication doses, if they had the relevant clinical information to do so. However, the majority of pharmacists believe physicians are opposed to such sharing of clinical data (quote 7.3, Table 2). They noted that physicians often want to preserve their professional jurisdiction by refusing to share all clinical information about a patient, such as treatment indication and laboratory test results.

\section{Execution of prescriptions}

The majority of pharmacists interviewed believe that e-Rx technology can contribute to their professionalization by facilitating the prescription execution process. One possible mechanism for this effect is process automation, which can reduce the amount of time spent on executing prescriptions. 
Table 1. Model of the effects of e-Rx on the professionalization of community pharmacists

Mechanism
generating the
effect Description

1. Analytical capacity

2. Dissemination of knowledge

3. Integration of process tasks

4. Process automation

5. Elimination of intermediaries
E-Rx can facilitate the pharmacist's decisionmaking in two ways:

1) clinical information about the patient is more detailed and circulates more rapidly between physicians and pharmacists. The information transmitted to the community pharmacist is more complete than that contained in handwritten prescriptions (e.g., treatment indication, dosage changes and stop orders);

2) expert software is available to the physician. This software can systematically analyze the information and check for alerts in the patient's record.

E-Rx can increase the quantity of information available to the physician at the time of prescribing by giving the physician access to: 1) clinical data (e.g., drug monographs, protocols, practice guides and treatment algorithms);

2) economic data (e.g., medication costs and insurance coverage).

E-Rx can improve coordination between the different tasks in the medication management process, which are split between physicians, pharmacists and other professionals.

All of the patient's clinical information can be combined in a single database, enabling a "virtual" bridging of the gap between professionals. E-Rx represents a first step toward achieving this integration of the activities of the physician and pharmacist both of whom are involved in medication management-because it makes them converge on a single patient file.

E-Rx can eliminate human-mediated operations in the process. It could lead to the automation of two operations during the prescription execution process:

1) entry of prescriptions in the pharmacist's computer system is automated due to electronic transmission. Therefore, the entry of prescriptions by the assistant or pharmacist is more rapid;

2) prescription interpretation by the assistant or pharmacist is more rapid because the physician uses a standard form for all prescriptions.

E-Rx can eliminate intermediaries in the transfer of information between the physician
Potential effects on the professionalization of community pharmacists

$\uparrow$ professionalization due to improvements in the exercise of the pharmacist's professional judgement during prescription execution.

$\uparrow$ professionalization due to a decrease in prescription errors and a resulting decrease in the amount of time spent on prescription execution.

$\downarrow$ professionalization due to an increase in the autonomy of physicians when prescribing medications and evaluating patients.

$\uparrow$ professionalization due to a decrease in prescription errors and a resulting decrease in the amount of time spent on prescription execution.

$\downarrow$ professionalization due to an increase in the autonomy of physicians when prescribing medications.

$\uparrow$ professionalization due to the facilitation of the pharmacist's involvement in different tasks of the medication management process (e.g., evaluation and follow-up).

$\uparrow$ professionalization due to a decrease in problems associated with handwritten prescriptions (e.g., illegible handwriting and incomplete information), and a resulting decrease in the amount of time spent on prescription execution.

$\uparrow$ professionalization due to a decrease in the problems associated with information 
Table 1

Continued

6. Tracking capability

7. Informational capability and pharmacist. Traditionally, the patient has served as an intermediary but this is no longer required because the pharmacist has direct access to the relevant clinical information in electronic format.

E-Rx can enable the pharmacist to monitor medication use and locate prescriptions in the computerized database in real time to determine their status. The handwritten prescription, which is passive and volatile, becomes an active prescription that leaves traces that can be followed in the patient's file.

E-Rx can also provide the pharmacist with a complete history of prescriptions made by the physician, whether or not the patient provides this information. The pharmacist can verify what was prescribed against what the patient requested.

E-Rx makes it possible to collect and analyze large data sets on medication management processes and patient populations. Prescriptions become not only retraceable, but also a source of basic data on physician prescribing practices (or prescription profiles), the management processes of pharmacists and patient consumption profiles. transmission by the patient (e.g., unknown, imprecise or incomplete information).

$\uparrow$ professionalization due to an increase in the pharmacist's ability to intervene with patients in order to manage treatment compliance. $\uparrow$ professionalization due to pharmacists now being able to carry out population-level analyses on their physician and patient clienteles (not only for prescriptions dispensed in a pharmacy, but also for the entire catchment population).

Table 2. The main effects of e-Rx technology on the professionalization of community pharmacists

\section{Mechanism generating the} effect

1. Analytical capacity of the pharmacist
1) improves the capacity of pharmacists to exercise their professional judgement when verifying prescriptions ( $\uparrow$ professionalization)

2) increases the relevance and meaningfulness of pharmacists' interventions with patients ( $\uparrow$ professionalization)
1) We could optimize treatments and make therapeutic choices that cost much less for the client and the public health system. When the pharmacist knows the treatment indication, he can do an amazing job-with respect to public costs, treatment safety and first treatment indication. (PHM3)

2.1) It avoids having to ask [the patient] extra and useless questions. Do you have a urinary tract infection or a sore throat? It avoids putting you and the patient in an uncomfortable position. (PHM9)

2.2) The other thing that I liked [about e-Rx] was that at least we had the indication. When we have the indication, we have a good basis from which to start explaining things to the patient. Because just think of all the conditions that beta blockers can be used to treat! And you can really get off track if you assume the medication is for this or that condition. So it's more reassuring for the patient. (PHM12) 
Table 2 Continued

2. Analytical capacity of the physician and dissemination of knowledge
3) increases the relevance and meaningfulness of pharmacists' interventions with prescribers ( $\uparrow$ professionalization)
4) reduces the number of clinical problems associated with prescriptions, thereby facilitating the pharmacist's verification of prescriptions and reducing the amount of time spent on prescription execution ( $\uparrow$ professionalization)
3.1) I will be able to make better suggestions to physicians, and also be more relevant in my interventions. And I won't have to bother them as much for nothing. (PHM4)

3.2) Having more information can help us make better decisions, or at least make suggestions. If we don't have the treatment indication, it's difficult to phone or send a message to the physician saying that it would be better if she made this or that change. Because we are going on assumptions. But if we have a more detailed file, we can see where we're going and give better advice. (PHM11)

4.1) There is less risk of observing an interaction down the line because [e-Rx] stops the doctor from prescribing a product that could cause an interaction. This would save the pharmacist a lot of time. If we could always reach them immediately when we observe a problem, it would be quick: when it is the same day, it takes three minutes to call. But often we have to phone five or six times before we reach anyone. (PHM12)

4.2) It allows [the physician] to rapidly detect any interactions and then make the right prescription. It slows us down when we have to phone the physician because there is an interaction on the prescription. (PHM6)

5) increases the physician's autonomy in managing the pharmacotherapy of patients ( $\uparrow$ or $\downarrow$ professionalization)
5.1) Perhaps [physicians] will call [us] less for summaries of records because they will already have them. This could save time, both for us and them. That would be great! (PHM12)

5.2) If physicians have any questions, instead of phoning us they will be able to get the information from the system. This will make things easier for everybody. (PHM11)

5.3) It could go either way. Some physicians could say, "fine, I really don't need to talk to the pharmacist anymore”, or "I don't need the pharmacist's advice anymore because I have a little machine that does the pharmacist's work". Or, physicians could say that this is going to be an additional tool for communicating with the pharmacist. I would say both of these reactions are valid. (PHM10)

Limitations of expert software

5.5) [Expert software] is useless as an intervention. [The physician] is going to call the pharmacist and say, "I have an interaction; what do I do about this?" (PHM3)

5.6) I don't think a machine can ever replace the pharmacist. Nor can a book. There are just too many different clinical aspects to consider in drug therapy. It will certainly help, for example in limiting the number of basic interactions, that's for sure. But they are not always clinically significant. I think there will always be only one specialist in medication use, and that is the pharmacist. I have studied just that for four years! There's just no doubt about it! No manual or 
Table 2 Continued

3. Integration of process tasks
4. Process automation
6) improves the physician's understanding of the pharmacist's role ( $\uparrow$ professionalization)

7) improves the capacity of pharmacists to exercise their professional judgement, enabling them to manage patients proactively (e.g., treatment decisions and follow-up) ( $\uparrow$ professionalization) software can replace that. I hope that it will not stop [physicians] from consulting us. (PHM4)

6) Because the physician is probably going to discover everything that pharmacists are already doing in their work. Again, I think that many physicians think we just take the prescription and count pills. [With $\mathrm{e}-\mathrm{Rx}$, physicians] are going to see interactions and they are going to start realizing that we've been checking this for 10-15 years. (PHM12)

7.1) Perhaps eventually this system could also add new lab results to the system. It could all be integrated. I know that companies are coming out with tracking software for lab data. It would be good if this data was shared for follow-up. You could automatically download it. If the [computer system] integrates lab data and diagnostic information, the role of the pharmacist [could be] even more important, more proactive. (PHM2)

7.2) The system could influence the pharmacist's role to the extent that [information] is also available to him, to allow him to play his role. (PHM4)

Resistance from physicians

7.3) We could have access to additional data that physicians refuse to provide. They do not want the diagnosis to be transmitted to the pharmacist. But I think this tight guarding of information will, with time, gradually fall by the wayside as the barriers between professions break down. Because professionals are going to realize that they can't keep working in isolation. (PHM10)

8) facilitates the entry of prescriptions, thereby reducing the amount of time spent on prescription execution ( $\uparrow$ professionalization)
8.1) This could make our work easier in terms of entering data in the computer - it would all be computerized. [It is] faster. Again, I am thinking about clinical practice, about spending my time not just on the computer. Of course, I have to do it. But the more time I have for my patients, the better. (PHM4)

8.2) It's just going to be faster. [...] It will free up technical time that the pharmacist will then be able to spend on real practice time. (PHM7)

8.3) If there are several medications on a prescription, just entering the number [of the prescription] and having everything recorded [in the patient's file] will save us a lot of time. If there is only one medication, it doesn't change things much. But for more than two or three medications, it saves us a fair bit of time. (PHM12)

Depends on the quality of the e-Rx entered by the physician

8.4) The fact that when I download it, everything automatically appears? But I have to change the posologies; they are never right. They are never written like they are supposed to be. (PHM8)

8.5) Again, the physician has to have entered [the prescription] correctly. Because when a hundred thousand arrive every hour in my system, there could 
Table 2 Continued

5. Eliminates the patient as the intermediary between the physician and pharmacist
9) facilitates the interpretation of prescriptions, thereby reducing the amount of time execution ( $\uparrow$ professionalization) spent on prescription
10) improves the relevance and meaningfulness of interactions between pharmacists and physicians ( $\uparrow$ professionalization) also be a hundred thousand errors to correct every hour! (PHM7)

9.1) I like electronic prescriptions better than handwritten ones, which are illegible. [It helps reduce] the number of problems with drug format, drug names that resemble each other and drug concentrations we can't read. Sometimes we can't even read the name of the physician, or the signature. (PHM9)

9.2) There is less time wasted trying to decipher illegible prescriptions. It doesn't happen everyday, but it happens fairly often. (PHM7)

9.3) Whenever [the prescription] is handwritten, there is a much higher risk of making errors when we read it. Of course, when we are unsure, we call [the prescriber]. But sometimes we don't have any doubts but we make an error. This happened to me yesterday. I didn't have the least bit of doubt so I didn't even think of calling. I ended up calling for some other reason. If it had been typewritten or printed there would have been no uncertainty. We can be sure. (PHM4)

Depends on the quality of the format of the e-Rx

9.4) We had problems at first. Some of the dosage schedules were hard to understand because they used a code that we don't usually use on prescriptions. For awhile it was hard to understand. Because basically you see that it is calculated from an algorithm - you check the boxes and it generates a document. It didn't give the usual bid or qd or $1 \mathrm{x} /$ day [but instead used some unusual notation]. (PHM2)

10.1) There will be no more calling [the physician] to ask, "Is this a 1 or a 2 that you wrote here?" There will only be more relevant interactions. And at the end of the day, the patient is the winner. (PHM2)

10.2) There will be fewer calls by pharmacists about illegible prescriptions. And less errors in the long run. Because many pharmacists do not dare call physicians. But you always have to doublecheck. (PHM7)

10.3) No doubt there will be fewer unnecessary calls. Bothering [the physician] when you can't read the prescription, that's what annoys them the most. And then when you telephone with a real question, they are fed up with getting so many calls. (PHM6)

10.4) Since it will decrease the number of interventions, when there is an intervention, it will be more relevant. So I think that when we talk, it will be really worth it. (PHM12)

11) Improves the quality of information transmitted to the pharmacist, thereby facilitating prescription verification and improving the capacity of pharmacists to exercise their
11.1) It often happens that when we call the physician to check a dosage that was represcribed, it is not the same strength, which the patient doesn't know. And filling a prescription is just that: a lot of time spent checking that it is really what the physician wanted to prescribe. If the file is there, we can concentrate on other things and do other pharmaceutical tasks. 


\section{Table 2 Continued}

professional judgement during prescription verification

( $\uparrow$ professionalization)
Maybe this could be a big advantage. (PHM4) 11.2) For us, it gives a little more information on medications that are stopped. Often physicians tell [their patients], but don't indicate it on the prescription. Then they replace it with another blood pressure lowering drug. So we have to figure it out: was the other drug stopped, is this an addition? For patients who know, it's okay. But some patients aren't sure. So I found that it gave me more information. (PHM11)

11.3) The physician does not always give a clear diagnosis to the patient. And sometimes it is given, but rapidly, and the patient doesn't understand. The patient arrives at the pharmacy and we have to depend on what he tells us. So is it pharyngitis, bronchitis or pneumonia that he has? We don't have the information, and I think a system like this can help us by providing the information, and help the patient.

(PHM10)
According to half of the pharmacists interviewed, the automation of prescription entry saves time, especially when the prescription includes more than three medications (quotes 8.1 to 8.3, Table 2). Some pharmacists felt that such time savings were not significant and that manual entry was just as fast. However, most pharmacists felt that the standard format used in the electronic prescription can reduce the amount of time spent on prescription execution because it leads to the automation of prescription interpretation (quotes 9.1 to 9.3, Table $2)$. This standardization can also decrease the number of telephone calls to the physician for clarification purposes, and therefore increase the relevance and meaningfulness of pharmacist/physician interactions (quotes 10.1 to 10.4, Table 2).

Some pharmacists noted that the potential positive effects of e-Rx were dependent on how it is used by physicians. Some pharmacists noted errors on prescriptions generated by the system due to, for example, a lack of correspondence between the format of the electronic prescription and that used in their pharmacy, or the physicians' inexperience with the computerized system (quotes 8.4, 8.5 and 9.4, Table 2).

In this case, the automation of prescription entry would not necessarily lead to a time savings vis-à-vis the traditional prescription execution process since the pharmacist must verify e-Rx entries made by the physician (quotes 8.4 and 8.5, Table 2). Moreover, from a legal and deontological point of view, the pharmacist is required to validate the details of the prescription (manufacturer, format, dosage, etc.) for the product that he actually serves to the patient. The amount of time spent on prescription execution can therefore actually increase when the pharmacist has to correct entries made by physicians, rather than those made by a pharmacy technician from a written prescription who is more familiar with the pharmacy's usual practices.

E-Rx can also facilitate the prescription execution process by decreasing the number of prescription errors. The majority of the pharmacists mentioned that clinical problems such as drug interactions, allergies and incorrect doses can decrease due to improvements in the analytical capacity of the physician and the dissemination of knowledge (quotes 4.1 and 4.2, Table 2). In general, the pharmacists appreciated the fact that the physician can access the patient's pharmacotherapeutic record, knowledge databases on medications and expert software. A number of pharmacists predicted that physicians would not have to call them as much, which they saw as positive (quotes 5.1 and 5.2, Table 2). Only two pharmacists saw this technology as a threat to their role as the medication specialist. However, they did not see this threat as a given, but rather as a possibility (quote 5.3, Table 2). In the same vein, some pharmacists stressed the limitations of the expert software with respect to their professional expertise, which explains why they did not feel any great threat from e-Rx (quotes 5.4 and 5.5, Table 2). The limitations of expert software, according to them, simply reinforce their importance as medication specialists. Finally, some pharmacists predicted that when physicians actually use the expert software or access a patient's complete 
pharmacotherapeutic record, as is done at the pharmacy, they will better understand the important role of the community pharmacist in medication management (quote 6, Table 2).

Two of the seven mechanisms by which e-Rx can potentially modify the practice of pharmacists, tracking capability and informational capability, were not mentioned by the pharmacists as influences on their professionalization. First, the tool did not allow the pharmacist to consult a patient's prescription record without the patient's authorization (for confidentiality reasons). Therefore, none of the pharmacists considered what effects the tracking capability can potentially have on their involvement in monitoring patient treatment compliance. Moreover, the pharmacists did not appear to envision any new possibilities related to informational capability, probably because their usual patient record management software already enables them to do such analyses on their clientele.

\section{DISCUSSION}

Our results suggest that e-Rx technology can have a positive effect on the professionalization of community pharmacists by enhancing the quality of information available to them and by improving the quality of prescriptions they execute. These two elements can favour the professionalization of community pharmacists by improving the capacity of pharmacists to exercise their professional judgement, by decreasing the amount of time spent on prescription execution, and by reshaping the image of the community pharmacist. We will now address each of these themes in more detail.

\section{E-Rx and the capacity of pharmacists to exercise their professional judgement}

Pharmacists are trained to evaluate a patient's pharmacotherapy based on the patient's condition (e.g., diagnosis, laboratory test results, allergies, life style and preferences). Community pharmacists are required by standards of practice to analyze the patient's pharmacotherapeutic record when executing or renewing a prescription. Currently, the information available to help them when exercising their professional judgement is limited. Often verifying a prescription is just a technical task, limited to validating its legal conformity. E-Rx can provide pharmacists with more precise and complete clinical information, thereby enabling them to exercise their clinical judgement more appropriately during a pharmacotherapeutic evaluation.

Pharmacists also seem ready to participate more actively in managing the pharmacotherapy of patients if they have access to the relevant clinical information. Our results confirm what several studies have already shown, namely that access to clinical information is a prerequisite to facilitating a broadening of the community pharmacist's role (2931). E-Rx, through the mechanism of integration of process tasks, can enable pharmacists to access this information. In this sense, e-Rx is a collaborative technology that facilitates the creation of a team composed of professionals working at different sites. Thus, rather than being confined to just the prescription execution process step, community pharmacists can participate in all aspects of the medication management process in the community (see Figure 1), from patient evaluation to medication use by patients, in accordance with their pharmacotherapeutic skills. In other words, the pharmacist can be a member of the clinical team, while still remaining in the pharmacy and overseeing the distribution of medications. It turns out that e-Rx can be a technology that paves the way for a greater professionalization of community pharmacists.

\section{The quality of e-Rx and prescription execution}

Our results show that e-Rx technology can facilitate the prescription execution process by improving the quality of prescriptions. The standardization of information contained in an e-Rx should limit the technical problems associated with handwritten prescriptions and enable the pharmacist to reduce the time currently spent on clarifying and completing these prescriptions. The validation of the information contained in the e-Rx by expert software, based on the patient's up-to-date pharmacotherapeutic record, can reduce the number of problems that community pharmacists encounter on prescriptions. This result confirms the findings of Buurma et al. (32), namely that the main determinant of community pharmacist interventions with prescribers and patients is the type of prescription (handwritten versus printed), irrespective of the characteristics of the prescription (e.g., number of medications or types of medications), the patient (e.g., pathology or age), or the prescriber. Thus, e-Rxs would be easier, and require less time, to execute than handwritten prescriptions. Given that pharmacists must intervene to clear up problems for approximately $2 \%$ of prescriptions they execute $(32,33)$, e-Rx technology can represent a significant gain in productivity.

In addition, the electronic transmission of prescriptions can facilitate the prescription verification process at the pharmacy. However, our results show that these benefits only arise if the information given on the e-Rx is consistent with that in the pharmacy's computer system and with the pharmacist's usual practices. If it is not, it can simply displace the task of the community pharmacist, from verifying the handwritten 
prescription to verifying the prescription entered by the physician. Some studies on electronic prescribing have noted this displacement. For example, Murray et al. (21) looked at the effects of e-Rx technology on the community pharmacist's work process pre- and post-implantation of the technology. They found that pharmacists spent $45 \%$ more time correcting problems associated with prescriptions transmitted electronically than they did handwritten prescriptions. Furthermore, managers of hospital pharmacies reported that up to $50 \%$ of prescriptions generated with e-Rx technology had to be cancelled by the pharmacist and redone in the computer system (34). This doubles the work and does no free up the pharmacist's time for activities of a clinical nature.

Some pharmacists also pointed out that there is no guarantee that the quality of prescriptions will improve with e-Rx technology. Even if the problems associated with handwritten prescriptions are eliminated, other problems could arise, such as inconsistent dosage sizes, frequencies, or errors in the selection of the drug. Several studies have shown that in the hospital context, e-Rx technologies can indeed generate these types of errors on prescriptions $(35,36)$. Verification by the pharmacist therefore becomes even more important, and more time-consuming (37). Thus the effects of e-Rx on the community pharmacist's execution of prescriptions depend on both the characteristics of the technology, such as flexibility and compatibility with the pharmacist's computer system, and on how the technology is used by prescribers. E-Rx can in fact slow down the prescription execution process and take pharmacists away from their clinical activities. In the field of medical informatics, numerous experts recognize that the majority of $\mathrm{e}-\mathrm{Rx}$ systems need further refining $(15,38)$. If community pharmacists want this technology to contribute to their professionalization, they need to collaborate in this development in order to ensure that systems marketed are well-adapted to their needs and practices (39).

\section{Prescription execution and the professionalization of community pharmacists}

Professional services are difficult to implement in any sustainable way in community pharmacies when there is a shortage of manpower coupled with an increase in the consumption of prescription medications $(30,31,40-42)$. To handle this challenge, pharmacists are looking for solutions that, like e-Rx, will decrease the time spent on prescription execution. However, there is no guarantee that an increase in the efficacy of prescription execution will translate into an increase in the amount of time spent on clinical activities. Two studies, carried out over twenty years ago, have examined the computerization of community pharmacies and tried to understand how this would alter the activities of community pharmacists (43, 44). They found that after the system was implemented, pharmacists spent less time on technical activities but not more time on professional activities. So even if the time spent on prescription execution decreases, we cannot know how pharmacists will use this newly available time. They may, for example, simply increase the number of prescriptions processed, without modifying in any significant way their current activities. Consequently, any mechanisms that act to facilitate prescription execution will create an opportunity for the restructuring of the community pharmacist's work, but what direction this transformation will take cannot be known with any certainty.

\section{E-Rx and the image of the community pharmacist}

To facilitate the implementation of professional services in community pharmacies, collaboration with prescribing physicians is essential. However, it has been widely documented that most physicians are hesitant about allowing community pharmacists to participate in any areas of pharmacotherapeutic management beyond the executing of prescriptions and the giving of advice and recommendations (6, $8,45-48)$. It would seem that physicians do not know what to expect from community pharmacists, and the commercial context that structures community pharmacy practice leads to the community pharmacist being seen as more of a shopkeeper than a health professional, which accentuates this ambiguity $(14,49)$. Several studies have shown that the key to fostering collaboration is trust, which requires that collaborators recognize each other's role and respect each other $(50,51)$. Certainly, our results suggest that e-Rx can have an impact on both of these elements.

First of all, e-Rx can help demystify the professional role of the community pharmacist for the physician by making the information and tools that community pharmacists are already using available to the physician. The physician, by trying out the expert software and seeing firsthand the complexity of patient pharmacotherapeutic profiles, which are based on the real behaviour of patients (e.g., treatment compliance and multiple physician involvement), will be able to better understand the community pharmacist's role and therefore how collaboration could be useful. This democratization of expert medication knowledge can be perceived as a threat by community pharmacists. However, the majority of pharmacists that we interviewed liked the fact that physicians can have access to this information. They also did not believe that computerized systems can replace their expertise on medicines, a reaction that has already been documented (52). 
Secondly, e-Rx can help strengthen the pharmacist's credibility as a specialist in medication use. Pharmacists' interactions with prescribers would become more relevant and meaningful through a number of different mechanisms. For example, the pharmacist would not need to make as many (often irritating) telephone calls to physicians seeking clarification. Moreover, pharmacists can use their clinical skills in pharmacotherapy to make interventions in accordance with information in the patient's record. Interactions between physicians and pharmacists would mainly concern patients and their use of medications, no longer just prescriptions and their characteristics. This should help pharmacists rid themselves of their image as merely the distributors of drugs.

\section{Strengths and limitations}

The results presented here need to be interpreted with caution due to the low level of e-Rx use in the pilot project on which these results are based. However, the pharmacists interviewed all possessed a good understanding of the system and all had executed prescriptions generated using the e-Rx technology. It should also be noted that this study was carried out in the early stages of electronic prescribing; perhaps the results will be different after more mature technologies are introduced and users have more fully mastered their use. On the other hand, users are often compelled to reflect on the experience at this stage, making it possible to discern phenomena that become less evident with habit. We believe that using a clear and detailed conceptual framework has allowed us to rigorously determine the potential effects as perceived by professionals who have tried the technology. Also, despite the limited number of pharmacists interviewed, their opinions were sufficiently convergent to make us confident in the strength and external validity of our results. In order to more fully understand this phenomenon, a greater number of pharmacists, practicing in a greater diversity of work environments, should be consulted. It would also be interesting to examine the perceptions of general practitioners and patients on this subject; this would help us better understand the direction that community pharmacy practice will take in the age of electronic prescribing.

\section{CONCLUSION}

This study enables us to better understand the perceptions of community pharmacists about the ways in which e-Rx technology could potentially influence their professionalization. Our results show that the technology of e-Rx can facilitate this process, mainly by improving the quality of clinical information available to the community pharmacist. E-Rx can also become a collaborative technology to the extent that it improves collaboration between community pharmacists and prescribing physicians. The automation of prescribing can also facilitate the prescription execution process for the pharmacist, but the specific effects are highly dependent on how compatible the technology is with the current practices of professionals. Finally, e-Rx can contribute to the professionalization of community pharmacists by acting not only on the medication management process in community pharmacy, but also on the image of the pharmacist. In short, this technology can enable pharmacists to solidify their role as a specialist in medication use by improving the relevance and meaningfulness of their interventions with prescribers and patients. In general, e-Rx proposes ways of working and communicating that were previously unimaginable. These new possibilities pave the way for transformations that can have a significant impact on the professionalization of community pharmacists. The results of this study also show that community pharmacists view the influence of e-Rx in a favourable light, believing it can be an ally in their professionalization.

\section{ACKNOWLEDGEMENTS}

We would like to thank the community pharmacists who took part in the interviews, as well as the following for their financial support: the interdisciplinary educational program, Analyse et évaluation des interventions en santé (AnÉIS); the Fond de la recherche en santé du Québec (FRSQ); and the Groupe de recherche interdisciplinaire en santé (GRIS).

\section{REFERENCES}

[1]. Denzin N. Incomplete professionalization: the case of pharmacy. in: Freidson E, Lorber J (eds), Medical men and their work. Aldine-Atherton, Chicago, 55-64, 1972.

[2]. Sleath B, Campbell W. American pharmacy: A profession in the final stage of dividing. J Social and Administrative Pharmacy, 15(4):225-40, 1998.

[3]. Eaton G, Webb B. Boundary encroachment: pharmacists in the clinical setting. Sociology of Health \& Illness, 1:69-89, 1979.

[4]. Hepler CD, Strand LM. Opportunities and responsibilities in pharmaceutical care. Am J Hosp Pharm, 47(3):533-43, 1990.

[5]. Cipolle RJ, Strand LM, Morley PC. Pharmaceutical care practice. 2nd ed., McGrawHill Companies, New York, 2004.

[6]. Gilbert L. To diagnose, prescribe and dispense: whose right is it? The ongoing struggle between pharmacy and medicine in South Africa. Current Sociology, 49(3):97-118, 2001.

[7]. Birenbaum A. Reprofessionalization in pharmacy. Soc Sci Med, 16(8):871-8, 1982. 
[8]. Edmunds J, Calnan MW. The reprofessionalisation of community pharmacy? An exploration of attitudes to extended roles for community pharmacists amongst pharmacists and General Practioners in the United Kingdom. Soc Sci Med, 53(7):943-55, 2001.

[9]. Berenguer B, La Casa C, de la Matta MJ, MartinCalero MJ. Pharmaceutical care: past, present and future. Curr Pharm Des, 10(31):3931-46, 2004.

[10]. Strand LM, Cipolle RJ, Morley PC, Frakes MJ. The impact of pharmaceutical care practice on the practitioner and the patient in the ambulatory practice setting: twenty-five years of experience. Curr Pharm Des, 10(31):3987-4001, 2004.

[11]. Christensen DB, Farris KB. Pharmaceutical Care in Community Pharmacies: Practice and Research in the US. Ann Pharmacother, 40(7):1400-6, 2006.

[12]. Jones EJ, Mackinnon NJ, Tsuyuki RT. Pharmaceutical care in community pharmacies: practice and research in Canada. Ann Pharmacother, 39(9):1527-33, 2005.

[13]. Maddux MS, Dong BJ, Miller WA, Nelson KM, Raebel MA, Raehl CL, et al. A vision of pharmacy's future roles, responsibilities, and manpower needs in the United States. American College of Clinical Pharmacy. Pharmacotherapy, 20(8):991-1020, 2000.

[14]. Hughes CM, McCann S. Perceived interprofessional barriers between community pharmacists and general practitioners: a qualitative assessment. $\mathrm{Br} \mathrm{J}$ Gen Pract, 53(493):600-6, 2003.

[15]. Bell DS, Cretin S, Marken RS, Landman AB. A conceptual framework for evaluating outpatient electronic prescribing systems based on their functional capabilities. J Am Med Inform Assoc, 11(1):60-70, 2004.

[16]. Corley ST. Electronic prescribing: a review of costs and benefits. Top Health Inf Manage, 24(1):29-38, 2003.

[17]. Kaushal R, Shojania KG, Bates DW. Effects of computerized physician order entry and clinical decision support systems on medication safety: a systematic review. Arch Intern Med, 163(12):1409-16, 2003.

[18]. Harrison MI, Koppel R, Bar-Lev S. Unintended Consequences of Information Technologies in Health Care An Interactive Sociotechnical Analysis. J Am Med Inform Assoc, 14(5):542-9, 2007.

[19]. Ash JS, Sittig DF, Poon EG, Guappone K, Campbell E, Dykstra RH. The extent and importance of unintended consequences related to computerized provider order entry. J Am Med Inform Assoc, 14(4):415-23, 2007.

[20]. Murray MD. Information technology: the infrastructure for improvements to the medication-use process. Am J Health Syst Pharm, 57(6):565-71, 2000.

[21]. Murray MD, Loos B, Tu W, Eckert GJ, Zhou XH, Tierney WM. Effects of computer-based prescribing on pharmacist work patterns. J Am Med Inform Assoc, 5(6):546-53, 1998.
[22]. Braveman H. Labor and Monopoly Capital: the degradation of work in the twentieth century. Monthly Review Press, New York, 1974.

[23]. Doolin B. Sociotechnical networks and information management in health care. Accting, Mgmt and Info Tech, 9:95-114, 1999.

[24]. Mclaughlin J, Webster A. Rationalising knowledge: IT systems, professional identities and power. Sociological Review, 46(4):781-802, 1998.

[25]. Davenport T. Process Innovation. Reingeneering work through information technology. Ernst and Young, Boston, 1983.

[26]. Davenport T, Short J. The new industrial engineering: information technology and business process redesign. Sloan Management Review:1127, 1990.

[27]. Tamblyn R, Huang A, Kawasumi Y, Bartlett G, Grad R, Jacques A, et al. The development and evaluation of an integrated electronic prescribing and drug management system for primary care. $\mathbf{J}$ Am Med Inform Assoc, 13(2):148-59, 2006.

[28]. Patton MQ. Qualitative analysis and interpretation. Chap. 8. in: Patton MQ (ed), Qualitative research and evaluation methods. 3rd ed., Sage Publications, Newbury Park, pp 431540, 2002.

[29]. Bell HM, Mcelnay J, hughes C, Woods A. A qualitative investigation of the attitudes and opinions of community pharmacists to pharmaceutical care. J Social and Administrative Pharmacy, 15(4):284-95, 1998.

[30]. Dunlop JA, Shaw JP. Community pharmacists' perspectives on pharmaceutical care implementation in New Zealand. Pharm World Sci, 24(6):224-30, 2002.

[31]. Odedina F, Segal R, Hepler C. Providing pharmaceutical care in community practice: Differences between providers and non-providers of pharmaceutical care. $J$ Social and Administrative Pharmacy, 12(4):170-9, 1995.

[32]. Buurma H, de Smet PA, van den Hoff OP, Egberts AC. Nature, frequency and determinants of prescription modifications in Dutch community pharmacies. Br J Clin Pharmacol, 52(1):85-91, 2001.

[33]. Westein MP, Herings RM, Leufkens HG. Determinants of pharmacists' interventions linked to prescription processing. Pharm World Sci, 23(3):98-101, 2001.

[34]. Inquilla CC, Szeinbach S, Seoane-Vazquez E, Kappeler KH. Pharmacists' perceptions of computerized prescriber-order-entry systems. Am J Health Syst Pharm, 64(15):1626-32, 2007.

[35]. Ash JS, Sittig DF, Dykstra RH, Guappone K, Carpenter JD, Seshadri V. Categorizing the unintended sociotechnical consequences of computerized provider order entry. Int $\mathrm{J}$ Med Inform, 76(S1):S21-S7, 2007.

[36]. Koppel R, Metlay JP, Cohen A, Abaluck B, Localio AR, Kimmel SE, et al. Role of computerized physician order entry systems in facilitating medication errors. JAMA, 293(10):1197-203, 2005. 
[37]. Fair MA, Pane F. Pharmacist interventions in electronic drug orders entered by prescribers. Am J Health Syst Pharm, 61(12):1286-8, 2004.

[38]. Wang CJ, Marken RS, Meili RC, Straus JB, Landman AB, Bell DS. Functional Characteristics of Commercial Ambulatory Electronic Prescribing Systems: A Field Study. J Am Med Inform Assoc, 12(3):346-56, 2005.

[39]. Estellat C, Colombet I, Vautier S, Huault-Quentel J, Durieux P, Sabatier B. Impact of pharmacy validation in a computerized physician order entry context. Int J Qual Health Care:mzm025, 2007.

[40]. Brown CM, Barner JC, Shepherd MD. Issues and barriers related to the provision of pharmaceutical care in community health centers and migrant health centers. J Am Pharm Assoc, 43(1):75-7, 2003.

[41]. Amsler MR, Murray MD, Tierney WM, Brewer N, Harris LE, Marrero DG, et al. Pharmaceutical care in chain pharmacies: beliefs and attitudes of pharmacists and patients. J Am Pharm Assoc, 41(6):850-5, 2001.

[42]. McDonough RP, JP R, JD C. Obstacles to the implementation of pharmaceutical care in the community setting. J Am Pharm Assoc, 35:87-95, 1998.

[43]. McKay AB, Sharpe TR, Smith MC, Jackson RA. Changes in traditional community pharmacist work patterns following computerization. Pharm Manage Comb Am J Pharm, 151(4):180-4, 90, 1979.

[44]. Moss RL, Pounders JN. Impact of computerization on dispensing time in an ambulatory-care pharmacy. Am J Hosp Pharm, 42(2):309-12, 1985.

[45]. Ranelli P, Biss J. Physician's perceptions of communication with and responsibilities of pharmacists. J Am Pharm Assoc, 40(5):625-30, 2000.

[46]. Sheppard C, Hunt A, Lupton C, Begley S. Community pharmacists in primary care: Prospects for pharmacist-doctor collaboration. J Social and Administrative Pharmacy, 12(4):181-9, 1995.

[47]. Muijrers PE, Knottnerus JA, Sijbrandij J, Janknegt R, Grol RP. Changing relationships: attitudes and opinions of general practitioners and pharmacists regarding the role of the community pharmacist. Pharm World Sci, 25(5):235-41, 2003.

[48]. Adamcik BA, Ransford HE, Oppenheimer PR, Brown JF, Eagan PA, Weissman FG. New Clinical Roles for Pharmacists - a Study of Role Expansion. Soc Sci Med, 23(11):1187-200, 1986.

[49]. Smith WE, Ray MD, Shannon DM. Physicians' expectations of pharmacists. Am J Health Syst Pharm, 59(1):50-7, 2002.

[50]. Brock KA, Doucette WR. Collaborative working relationships between pharmacists and physicians: an exploratory study. J Am Pharm Assoc, 44(3):358-65, 2004.

[51]. Zillich AJ, McDonough RP, Carter BL, Doucette WR. Influential characteristics of physician/pharmacist collaborative relationships. Ann Pharmacother, 38(5):764-70, 2004.
[52]. Novek J. Hospital pharmacy automation: collective mobility or collective control? Soc Sci Med, 51(4):491-503, 2000. 